\title{
Physical modelling of cohesive soil inherent variability: consolidation problem
}

\author{
Reza Jamshidi Chenari ${ }^{1 *} \mathbb{D}$, Ali Taleb ${ }^{1}$, Malahat Ghoreishi ${ }^{1}$ and Meghdad Payan ${ }^{2}$
}

\author{
${ }^{*}$ Correspondence: \\ Jamshidi_reza@guilan.ac.ir \\ ${ }^{1}$ Department of Civil \\ Engineering, Faculty \\ of Engineering, University \\ of Guilan, P.O. 3756, Rasht, \\ Guilan, Iran \\ Full list of author information \\ is available at the end of the \\ article
}

\begin{abstract}
The main goal of this study is to substantiate the heterogeneous soil modelling in the laboratory and to examine the effect of spatial variability on the soil consolidation properties. For this purpose, several heterogeneous soil physical models are constructed using nine homogeneous soil clusters, which are prepared by mixing variable proportions of kaolin and bentonite at a specific water content equal to the liquid limit of the mixtures. The index properties of different homogeneous kaolin-bentonite clusters are utilized to model the spatial variability of the heterogeneous soil models using the random field generation approach. The physical model contains different discrete cells where the variability is controlled by the specific realizations of a random field. The number of these realizations in the heterogeneous model is augmented by varying the liquid limit of the mixtures. The constructed physical models are then subjected to several one-dimensional consolidation stages using the oedometer apparatus and the influence of the heterogeneity of the soil sample on its hydraulic parameters is evaluated. The results show that the higher variability involved in the soil strata, expressed through the larger coefficient of variation and smaller correlation distance, leads to the increase in the hydraulic conductivity and coefficient of consolidation of the heterogeneous soil physical model. It is also revealed that the experimental modelling of soil spatial variability can shed light on the numerical simulation of random field theory presented in several previous studies.
\end{abstract}

Keywords: Kaolin-bentonite mixture, Consolidation, Heterogeneous physical model, Hydraulic conductivity, Coefficient of variation

\section{Introduction}

Soil, resulting from several physical, chemical, geological and geo-environmental phenomena, is a complicated structural material which is highly heterogeneous in nature in terms of mechanical properties. Most of the studies performed on the stability analysis of geo-structures are restricted to the deterministic approach framework, i.e. considering constant soil parameters within the stress influence area. However, inherent uncertainties are inevitable in any geotechnical problem and while their ignorance can lead to the undesired and inappropriate estimation of the soil properties, their consideration can result in more practical and realistic vision of the soil behaviour $[6-8,14]$. Thereby, due to the great amount of uncertainties involved in the accurate evaluation of the soil properties in the field, probabilistic analysis of geo-structures seems to be an impartible and essential component of the geotechnical design.

(c) The Author(s) 2018. This article is distributed under the terms of the Creative Commons Attribution 4.0 International License (http://creativecommons.org/licenses/by/4.0/), which permits unrestricted use, distribution, and reproduction in any medium, provided you give appropriate credit to the original author(s) and the source, provide a link to the Creative Commons license, and indicate if changes were made. 
Implementing well-established numerical modellings along with the incorporation of the random field theory and Monte Carlo simulations, innumerable studies have been performed throughout the literature regarding the introduction of uncertainty involved in the estimation of soil properties into the variety of geotechnical problems, including bearing capacity and settlement of shallow and deep foundations, slope stability and liquefaction hazards $[2,4,6-8,11-16,18-20,22]$.

Despite these comprehensive studies on the numerical simulation of soil spatial variability and due to the large amount of cost and time required to construct full-scale physical models, there exist very few studies in the literature on the experimental verification of the numerical models, inspired by the seminal contributions of Garzón et al. [9], Ghoreishi [10] and Taleb [21]. In an innovative and comprehensive study performed by Garzón et al. [9], random field theory was combined with the geotechnical centrifuge modelling in order to propose a novel technique for the construction of reducedscale soil models with controlled spatial variabilities. With such a broad applicability for the verification and calibration of the numerical simulations, this new methodology was observed to offer the possibility for the study of the effects of soil variability on the behavior of geo-structures. This novel approach was later employed by Ghoreishi [10] and Taleb [21] in separate studies to reconstitute the heterogeneous physical models for the characterization of their properties in direct shear apparatus.

Apart from these pioneer advances, to the best of authors' knowledge, there exists no study throughout the literature on the physical modelling of soil spatial variability along with the experimental evaluation of the consolidation properties of heterogeneous soil samples. In this study, random field theory concepts are implemented to reconstitute several heterogeneous soil physical models using the variable proportions of homogeneous kaolin-bentonite mixtures. In order to justify the use of the kaolin-bentonite mixtures for the heterogeneous sample construction, their physical and mechanical properties are thoroughly investigated through several index and oedometer experiments which are then utilized to simulate the spatial variability in the heterogeneous model. The constructed physical models are then subjected to several stages of consolidation process in a large scale consolidometer to examine the effects of heterogeneity and spatial variability on the consolidation and permeability parameters of the tested soils.

\section{Material used}

The primary soils used in this study are commercially available bentonite, kaolin, and colorants. Bentonite and kaolin, which were procured from a local supplier, have been selected in this study to virtually reconstitute heterogeneous samples in the laboratory. As defined by geologists, bentonite is a highly colloidal and plastic clay composed mainly of the mineral montmorillonite. It has high capability to absorb water and is widely used in the civil engineering projects as a barrier for landfills, slurry walls and grout curtains due to its small particle size, low permeability and high swelling potential. The reason behind using bentonite as one of the constituents lies on the fact that it offers versatility for heterogeneous sample construction as its properties varies with the moisture content. On the other hand, kaolin, commonly referred to as china clay, is a natural clay formed by rock weathering and consists of kaolinite, 


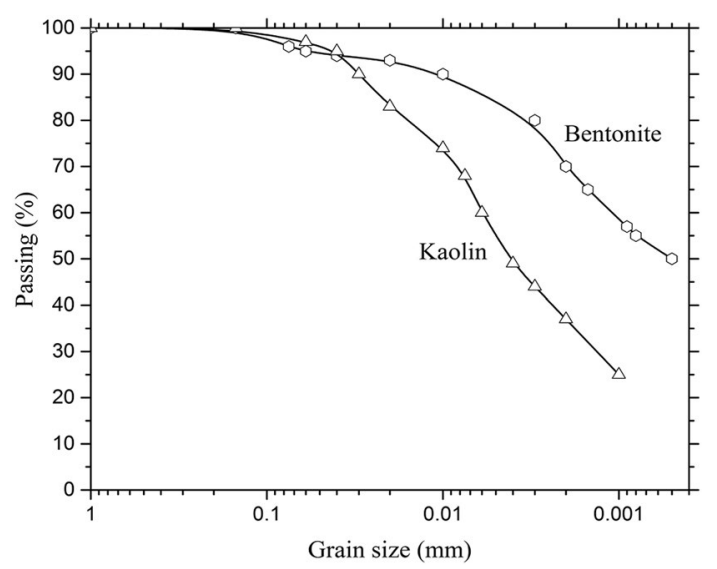

Fig. 1 Particle size distribution curves of the bentonite and kaolin used in this study

Table 1 Index properties of the kaolin and bentonite used in this study

\begin{tabular}{lll}
\hline Property & \multicolumn{2}{l}{ Range } \\
\cline { 3 - 3 } & Kaolin & Bentonite \\
\hline Material passing sieve\# 200 (\%) & $>90$ & $>95$ \\
Liquid limit (\%) & 50 & 346 \\
Plastic limit (\%) & 31 & 56 \\
Plasticity index & 19 & 290 \\
Maximum dry density $\left(\mathrm{g} / \mathrm{cm}^{3}\right)$ & 1.55 & 1.19 \\
Optimum moisture content (\%) & 25.08 & 42.74 \\
Specific surface area $\left(\mathrm{m}^{2} / \mathrm{g}\right)$ & 15 & 400 \\
\hline
\end{tabular}

quartz, mica, feldspar, illite and montmorillonite [17]. Kaolin, used as the other constituent of the heterogeneous samples, renders stability to mixture, thus enabling to achieve blends with higher strength or permeability. Different from bentonite, kaolin possesses a very low potential to absorb water [17]. On the other hand, similar to bentonite, it is also a low permeability clay; however, its permeability is higher than that of bentonite. Mixing bentonite with kaolin leads to enhance the strength properties and causes the compressibility characteristics of the mixture tending towards those of bentonite. In other words, bentonite, for its water affinity, is to be mixed with kaolin, representing less active material, to render a spectrum of blends which are utilized to reconstitute the so-called heterogeneous soil samples.

The grain size distribution curves of the kaolin and bentonite are presented in Fig. 1. According to the Unified Soil Classification System (USCS), they can be both classified as high plastic clays $(\mathrm{CH})$. Table 1 also presents the index properties of the clays used in this study. As can be seen in this table, bentonite has smaller particles with larger specific surfaces compared to kaolin, leading to the higher potential for swelling and plasticity which can be attributed to its affinity for water absorption. On the other hand, the kaolin particles can be compacted more than bentonite in a given volume, resulting in the higher maximum dry density for kaolin in a standard Proctor 


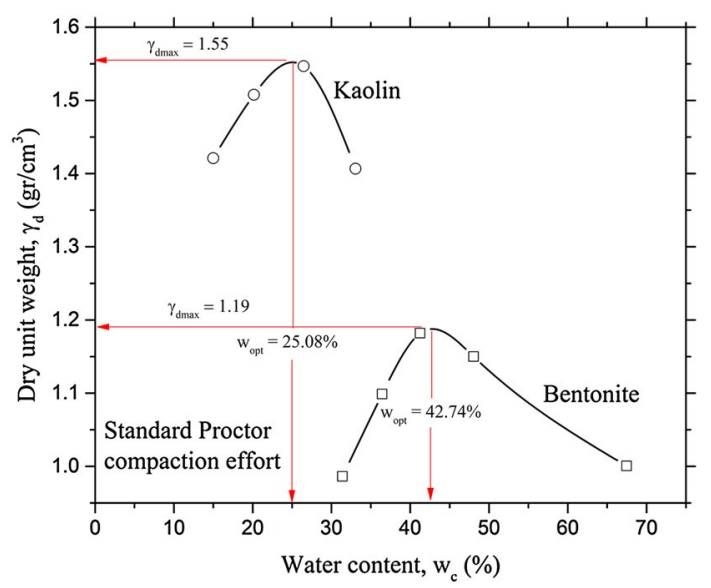

Fig. 2 Standard Proctor compaction curves of the kaolin and bentonite used in this study

Table 2 Index properties of the homogeneous soil mixtures

\begin{tabular}{|c|c|c|c|c|c|c|}
\hline Designation & Soil mixtures & LL (\%) & PL (\%) & $\mathrm{PI}(\%)$ & Classification & $G_{S}$ \\
\hline S1 & Kaolin & 50 & 31 & 19 & $\mathrm{CH}$ & 2.59 \\
\hline S2 & $80 \%$ kaolin $+11 \%$ bentonite $+9 \%$ colorant $^{\mathrm{a}}$ & 59 & 29 & 30 & $\mathrm{CH}$ & 2.69 \\
\hline S3 & $68 \%$ kaolin $+23 \%$ bentonite $+9 \%$ colorant $^{b}$ & 104 & 30 & 74 & $\mathrm{CH}$ & 2.64 \\
\hline S4 & $55 \%$ kaolin $+33 \%$ bentonite $+12 \%$ colorant $^{c}$ & 109 & 27 & 82 & $\mathrm{CH}$ & 2.61 \\
\hline S5 & $44 \%$ kaolin $+44 \%$ bentonite $+12 \%$ colorant $^{d}$ & 139 & 35 & 104 & $\mathrm{CH}$ & 2.65 \\
\hline S6 & $31 \%$ kaolin $+52 \%$ bentonite $+17 \%$ colorant $^{e}$ & 151 & 32 & 119 & $\mathrm{CH}$ & 2.72 \\
\hline S7 & $20 \%$ kaolin $+63 \%$ bentonite $+17 \%$ colorant $^{f}$ & 201 & 42 & 159 & $\mathrm{CH}$ & 2.75 \\
\hline S8 & $10 \%$ kaolin $+73 \%$ bentonite $+17 \%$ colorant $^{9}$ & 245 & 43 & 202 & $\mathrm{CH}$ & 2.78 \\
\hline s9 & Bentonite & 346 & 56 & 290 & $\mathrm{CH}$ & 2.80 \\
\hline
\end{tabular}

compaction test. Moreover, the optimum moisture content is higher for the bentonite compared to the kaolin (Fig. 2).

\section{Index properties of homogeneous soil clusters}

In order to justify the use of kaolin-bentonite mixture to fabricate different realizations of the heterogeneous samples, their respective physical and mechanical properties are obtained through various experimental techniques, which are summarized hereafter. The mechanical behavior for such soil samples was first expressed by Burland [3] who tried to propose a correlation between the geotechnical properties of reconstituted and natural clay samples. In this study, in addition to pure kaolin and pure bentonite, seven extra reconstituted homogeneous mixtures were prepared, as shown in Table 2. Colorant was employed to differentiate between the mixtures. All the mixtures were constructed in a water content equal to the liquid limit of the soil.

A set of index tests, including sieve analysis, hydrometery, Atterberg limit test, specific gravity and compaction test, were carried out on the homogeneous soil mixtures. As can be seen in Table 2, as the specific gravity of the mixture increases, its plastic behavior also shows ascending trend. In order to classify the soil mixtures, the well-established Casagrande plasticity chart along with the classification chart for the degree of swelling 
potential, originally developed by Dakshanamurthy and Raman [5], are adopted. As can be observed in Fig. 3, considering six different categories based on the soil liquid limit, Dakshanamurthy and Raman [5] tried to develop a criterion for the soil swelling potential. According to them, the mixtures S1 and S2 in this study have got high, while the other mixtures have got extra high degree of swelling potential. In addition, the first two mixtures are classified as medium and the other mixtures are categorized as very high in terms of the degree of swelling potential.

\section{Consolidation of homogeneous clusters}

Soil consolidation properties are essential parameters in the characterization of the soil mechanical behavior. The compressibility of a soil is often measured in a laboratory device known as an oedometer or consolidometer. The oedometer test is a useful experimental tool to determine the compressibility characteristics of the soils, including the compression index, $\mathrm{C}_{\mathrm{c}}$, coefficient of volume compressibility, $\mathrm{m}_{\mathrm{v}}$, hydraulic conductivity, $\mathrm{k}_{\mathrm{v}}$, and the coefficient of consolidation, $\mathrm{c}_{\mathrm{v}}$.

Kaolin-bentonite mixtures, typically used as a liner material in the landfills, are quite susceptible to consolidation settlement during the life of geo-structure. Therefore, the settlement analysis of these mixtures is a critical part of the geotechnical design of such structures. In this study, the influence of bentonite addition and consolidation pressure on the various consolidation parameters of the nine different kaolin-bentonite mixtures, introduced earlier, is evaluated.

\section{Experimental procedure}

In this study, several consolidation tests were performed to determine the consolidation behavior of nine kaolin-bentonite mixtures, as presented in Table 2. Tests were conducted on the samples of $60-\mathrm{mm}$ diameter and 20-mm thickness according to ASTM D2435-03 [1] using the standard oedometer. The cylindrical soil sample is confined inside a ring in order to prevent lateral strain. Porous stones are placed on both sides of the soil to permit the escape of water. The samples were prepared by mixing dry powders of kaolin, bentonite and sufficient amount of colorant, passing sieve No. 200 and the initial water content of the mixture was considered equal to its respective liquid limit. In order to avoid the friction between the ring and the soil inside, the wall of the ring

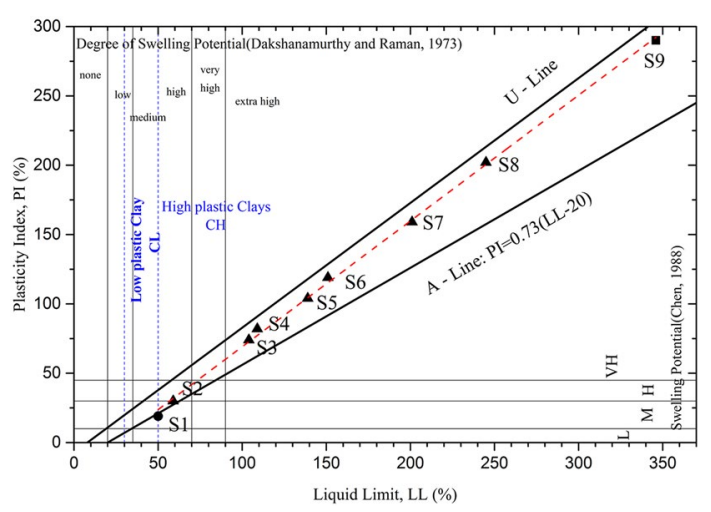

Fig. 3 Classification of the soil mixtures 
was smeared with the silicone grease. The vertical load is applied to the soil by means of weights applied through a lever system to the top of the soil. The amount of vertical settlement experienced by the soil as a result of the application of overburden pressure is measured by means of a dial gauge. In order not to let the sample to swell and to reach the stable condition, the samples were pre-pressurized by $7 \mathrm{kPa}$ before the start of the tests. The conventional testing technique consists of applying successive increments of load and observing the deflection after each increment until the movement ceases. In a saturated soil sample, the application of the vertical load results in the development of an excess pore pressure (equal to the vertical stress applied) within the soil which gradually dissipates as water is expelled from the soil through the porous stones. Movement of the soil continues until the excess pore pressure has been fully dissipated. In this study, all the samples, initially loaded to a pressure of $7 \mathrm{kPa}$, were subjected to the gradual incremental loading steps up to a maximum pressure of $200 \mathrm{kPa}$. Each pressure step was maintained on the samples for $48 \mathrm{~h}$ so as to let the excess pore water pressure dissipate. Based on the experimental results, there is a correlation between the percentage of bentonite in the mixture and the consolidation parameters, i.e. compression index $\left(C_{c}\right)$, coefficient of consolidation $\left(c_{v}\right)$ and the time for $50 \%$ of consolidation $\left(t_{50}\right)$.

\section{Results of consolidation tests on homogeneous clusters}

Figure 4 shows the one-dimensional compression curves for the reconstituted soil mixtures covering a wide range of plasticity. The results obtained from the consolidation experiments are also summarized in Table 3. Compression index $\left(C_{c}\right)$ for the samples was calculated as the slope of the straight line portion of the virgin e-log p curve as follows:

$$
C_{c}=\frac{e_{i}-e_{j}}{\log \left(p_{i} / p_{j}\right)}
$$

where $e_{i}$ and $e_{j}$ refer to the void ratios corresponding to the consolidation pressures of $p_{i}$ and $p_{j}$, respectively. Taylor's method was adopted in this study to obtain the coefficient of consolidation $\left(\mathrm{c}_{\mathrm{v}}\right)$. In order to check the reproducibility of the test results, a few samples (S1, S2 and S3) were chosen randomly, and the consolidation test was repeated. The

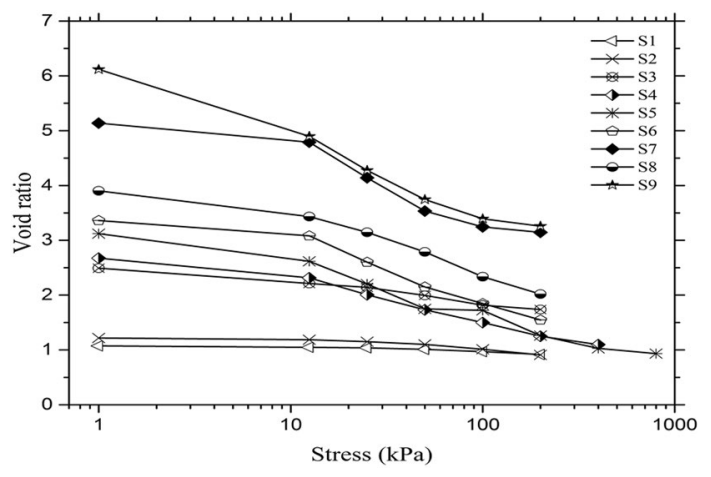

Fig. 4 Consolidation curves of the homogeneous soil mixtures 
Table 3 Summary of compressibility parameters of the homogeneous soil mixtures

\begin{tabular}{lllll}
\hline Designation & Initial void ratio, $\boldsymbol{e}_{\boldsymbol{o}}$ & $\begin{array}{l}\text { Compression } \\
\text { index, } \boldsymbol{C}_{\boldsymbol{c}}\end{array}$ & $\begin{array}{l}\text { Recompression } \\
\text { index, } \boldsymbol{C}_{\boldsymbol{r}}\end{array}$ & $\boldsymbol{c}_{\boldsymbol{v}}\left(\mathbf{m m}^{\mathbf{2} / \mathbf{s})^{\mathbf{a}}}\right.$ \\
\hline S1 & 1.07277 & 0.11860 & 0.01960 & 0.61955 \\
S2 & 1.21720 & 0.23080 & 0.03820 & 0.02174 \\
S3 & 2.49220 & 0.39450 & 0.04120 & 0.00251 \\
S4 & 2.10874 & 0.60370 & 0.04910 & 0.00143 \\
S5 & 2.17488 & 0.67700 & 0.04320 & 0.00138 \\
S6 & 3.36213 & 1.27420 & 0.04630 & 0.00119 \\
S7 & 5.13793 & 1.34740 & 0.06370 & 0.00111 \\
S8 & 3.90134 & 1.17520 & 0.10210 & 0.00062 \\
S9 & 6.11932 & 1.35910 & 0.07230 & 0.00043 \\
\hline
\end{tabular}

a Coefficient of consolidation at pressure $100 \mathrm{kPa}$

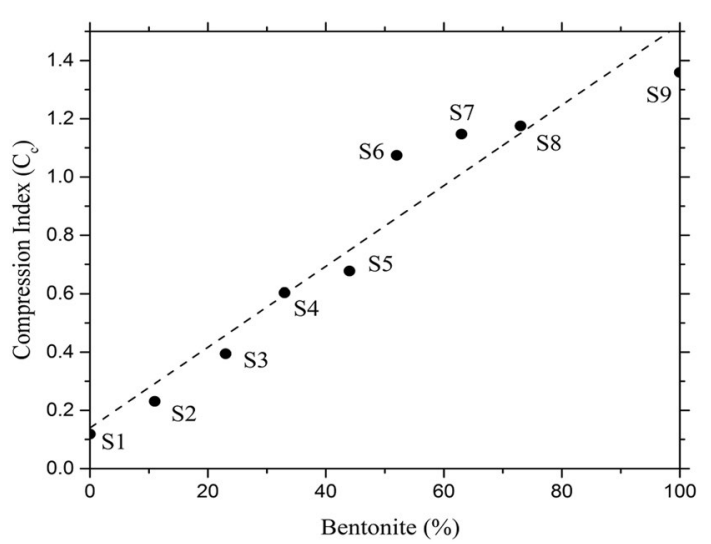

Fig. 5 Effect of bentonite content on the $c_{c}$ values

differences between the results of the two tests remained within $\pm 10 \%$ error which is quite acceptable.

Figure 5 shows the variation of the compression index with the percentage of bentonite in the mixtures. As can be observed in this figure, the increase in the bentonite percentage leads to the increase of the compression index of the kaolin-bentonite mixture which in turn implies a larger decrease in the void ratio of the mixture upon a particular stress increment. This means that a greater volume of water must be expelled from the soil, thus requiring a longer time period.

Figure 6 illustrates the variation of the coefficient of consolidation $\left(c_{v}\right)$ with the bentonite percentage used to prepare the samples. As shown in this figure, for some mixtures, $c_{v}$ values were found to increase with the increase in the consolidation pressure, indicating that the mixtures consolidate at a higher rate when subjected to a higher overburden pressure. The results also show that irrespective of the overburden pressure, the $\mathrm{C}_{\mathrm{v}}$ values decrease with the increase in the percentage of bentonite present in the kaolinbentonite mixtures. Accordingly, $t_{50}$ values for the mixtures were observed to increase with the increase in the percentage of bentonite in the mixtures.

Increasing the consolidation pressure, leads both permeability coefficient $\left(\mathrm{k}_{\mathrm{v}}\right)$ and coefficient of compressibility $\left(\mathrm{m}_{\mathrm{v}}\right)$ to decrease rapidly, resulting in a fairly constant value 


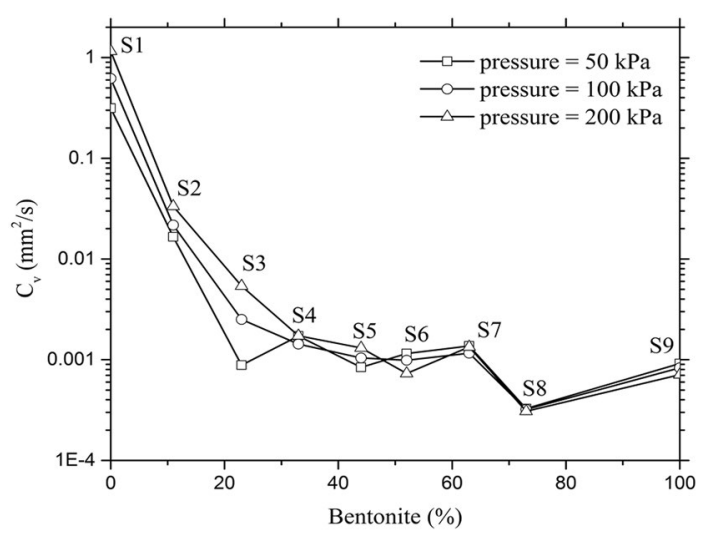

Fig. 6 Effect of bentonite content on the $c_{v}$ values

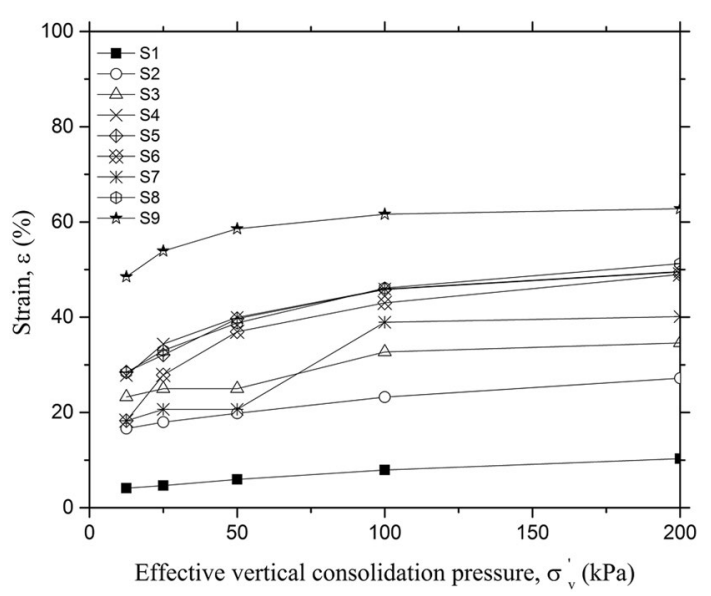

Fig. 7 Stress-strain relationship for the homogeneous kaolin-bentonite mixtures

for the ratio $\left(\mathrm{k}_{\mathrm{v}} / \mathrm{m}_{\mathrm{v}}\right)$ and, hence, constant $c_{v}$, over a wide range of consolidation pressures. However, results of this paper show that the variation of $c_{v}$ with consolidation pressure is considerable, rendering increasing trend for the mixtures in which kaolin is the predominant component and decreasing trend when the mixture contains greater amount of bentonite.

The stress-strain relationships obtained from the oedometer tests for the nine mixtures are shown in Fig. 7. As can be observed in this figure, while the pure kaolin has smaller strain among the investigated mixtures, the pure bentonite experiences the highest strain. Mixing the bentonite with the kaolin produces a mixture with the stiffness characteristics closer to those of bentonite. The kaolin exhibits a stiffer and more brittle behavior during the consolidation process compared to the bentonite which renders a semi plastic to plastic responses. This implies that the bentonite content plays an important role to produce a smoother and more plastic behavior of the mixture upon the load application.

Figure 8 shows the variation of the coefficient of consolidation with the applied stress for variable kaolin-bentonite mixtures. As shown in this figure, in the mixtures with a 

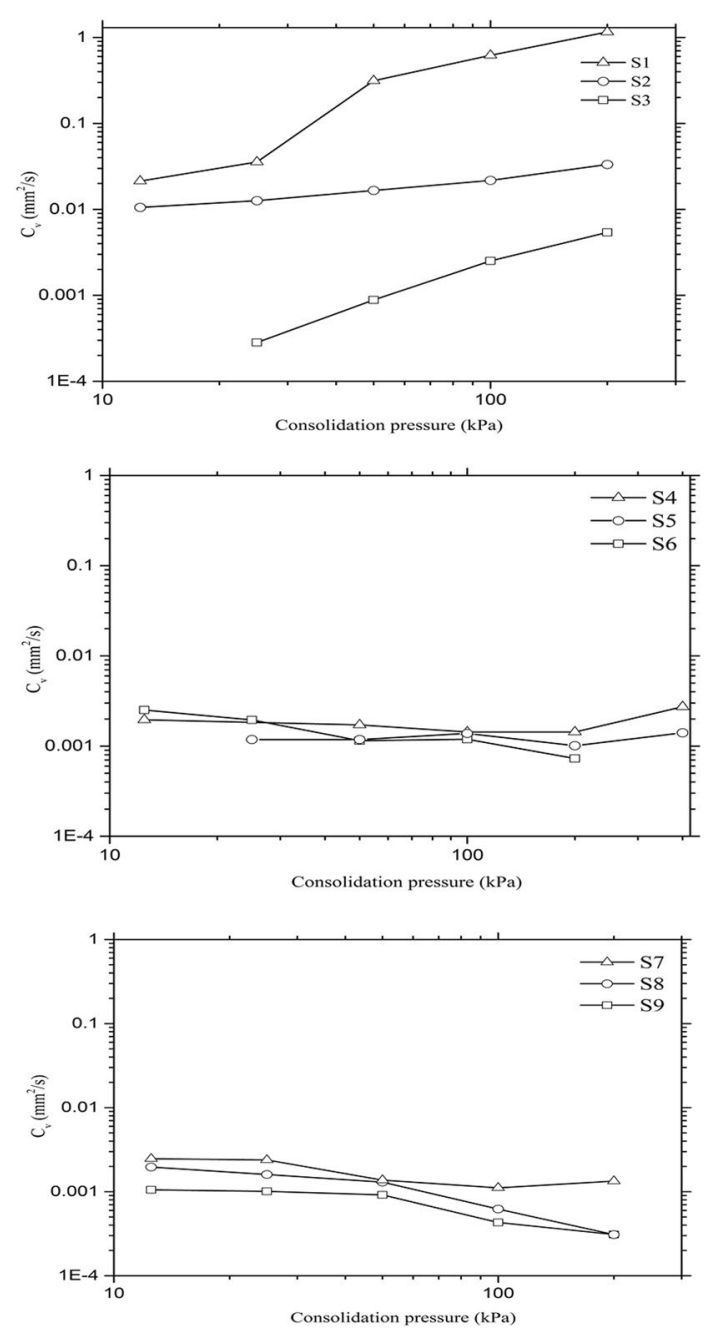

Fig. 8 Influence of stress level on the coefficient of consolidation

greater amount of kaolin, the coefficient of consolidation increases with the increase in the consolidation pressure. On the other hand, for mixtures in which bentonite is predominant, increasing the consolidation pressure leads to lower values for the coefficient of consolidation. For the mixtures S4 and S5, there exists no specific trend between the consolidation pressure and $\mathrm{c}_{\mathrm{v}}$. These observations can be attributed to the fact that the bentonite particles are smaller with larger specific area as compared to kaolin particles. In addition, there exist more negative charges on the surfaces of the bentonite particles, resulting in a considerable amount of repulsion forces among them. The repulsion forces due to the negative charges on the particle surfaces prevent the particles to become more compressed during the application of consolidation pressure. In other words, higher pressures, which cause the particles to approach each other, result in the increase of the repulsion forces, leading to the prolonged consolidation process.

Figure 9 shows the variation of the volume compressibility coefficient $\left(\mathrm{m}_{\mathrm{v}}\right)$ with the percentage of bentonite present in the mixtures. The compressibility coefficient is defined as the volumetric strain due to a unit increase in the stress level. In this 


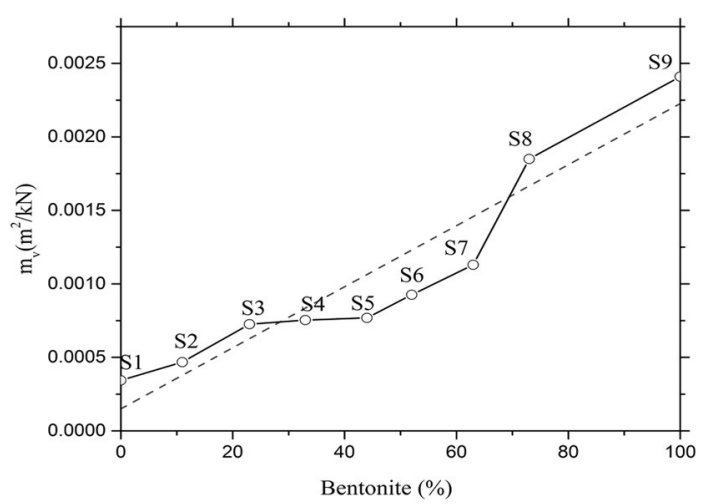

Fig. 9 Influence of bentonite content on the compressibility of soils

study, the void ratio of the samples at each pressure step is measured which in turn will be utilized to evaluate the volumetric strain through the following correlation:

$$
\Delta \varepsilon=\frac{\Delta V_{V}}{V_{S}+V_{V}}=\frac{\Delta V_{V} / V_{S}}{1+V_{V} / V_{S}}=\frac{\Delta e}{1+e_{i}}
$$

where $\Delta V_{V}$ is the change in the void volumes, $\mathrm{V}_{\mathrm{s}}$ and $\mathrm{V}_{\mathrm{v}}$ are the volumes of solid and void, respectively, $\Delta e$ is the change in the void ratio and $\mathrm{e}_{\mathrm{i}}$ is the initial void ratio of the sample.

As a result of an oedometer test, application of the overburden pressures $\left(\Delta \sigma_{v}^{\prime}\right)$ leads to the accumulated volumetric strain and consequently, the void ratio of the mixture changes. Accordingly, the coefficient of compressibility $\left(\mathrm{m}_{\mathrm{v}}\right)$ can be estimated by the following formula:

$$
m_{v}=\frac{\Delta \varepsilon}{\Delta \sigma_{v}^{\prime}}=\frac{\Delta e}{\left(1+e_{i}\right) \Delta \sigma_{v}^{\prime}}
$$

As shown in Fig. 9, $m_{v}$ values follow an ascending trend with the percentage of bentonite for the whole range of bentonite content. A greater compressibility leads to a greater decrease in the void spaces of the soil for a particular change in the overburden pressure. This means that a greater volume of water must be expelled from the soil sample which requires a longer period of time, resulting in a lower rate of consolidation.

Another parameter that influences the rate of consolidation is permeability. An increase in the permeability of the soil causes an increase in the rate of seepage flow. Having a greater rate of water expulsion from the soil, the pore pressure dissipates more rapidly, leading to a faster rate of consolidation. As can be observed in Fig. 10, the addition of a small amount of bentonite to the kaolin leads to a significant reduction of the permeability coefficient. Moreover, beyond about $30 \%$ of bentonite content, the coefficient of permeability of the mixture is almost the same as that of pure bentonite. In other words, this specific percentage is the threshold bentonite content for kaolin-bentonite mixtures after which bentonite portion of the mixture is controlling the soil behavior. 


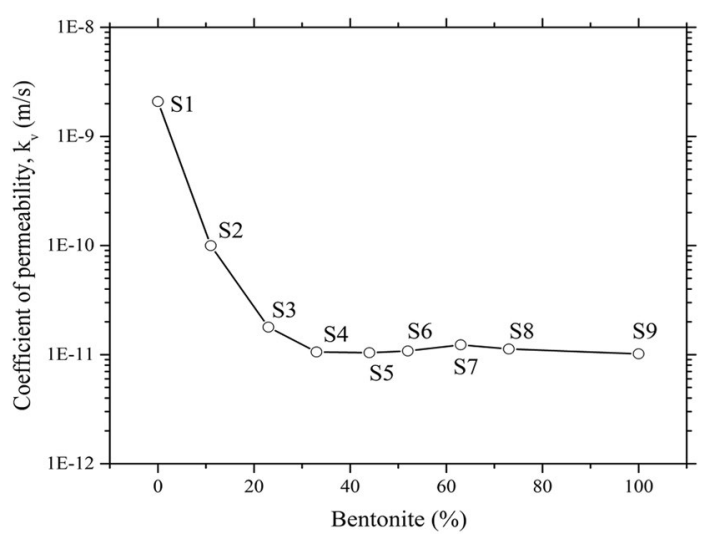

Fig. 10 Variation of coefficient of permeability $k_{v}$ with bentonite content

\section{Random field theory concepts}

Due to the large amount of uncertainties involved in the geotechnical engineering problems, probabilistic analysis of soil spatial variability in such analyses using random field theory would lead to more realistic and conservative outcomes. Inherent soil spatial variability refers to the general variation of soil properties among the points throughout the soil medium. Considering a typical soil property as the random variable, its geo-statistical parameters can be described through the mean $\left(\mu_{x}\right)$, variance $\left(\sigma_{x}^{2}\right)$, standard deviation $\left(S D\right.$ or $\left.\sigma_{x}\right)$, coefficient of variation (COV), which are defined as follow:

$$
\begin{aligned}
& \mu_{x}=\frac{1}{n} \sum_{i=1}^{n} x_{i} \\
& \sigma_{x}^{2}=\frac{1}{n-1} \sum_{i=1}^{n}\left(x_{i}-\mu_{x}\right)^{2} \\
& S D=\sqrt{\sigma_{x}^{2}}=\sigma_{x} \\
& C O V=\frac{\sigma_{x}}{\mu_{x}}
\end{aligned}
$$

where $x_{i}$ is the soil parameter of interest and $n$ is the number of sampling points throughout soil strata. The other important parameter for the characterization of probabilistic soil properties is the scale of fluctuation $(\theta)$, defined as the length beyond which no significant correlation in a specific soil property is observed. In other words, scale of fluctuation is the statistical parameter to characterize the degree of correlation of a soil property within the soil medium. The scale of fluctuation can be described in both the horizontal and vertical directions, denoted as $\theta_{\mathrm{h}}$ and $\theta_{\mathrm{v}}$, respectively.

In order to generate a random field for the soil properties, which are usually strictly non-negative, a log-normal distribution could be adopted. Accordingly, the soil property is defined as follows: 


$$
\xi(\tilde{X})=\exp \left(L \cdot \varepsilon_{\ln \xi(\tilde{X})}+\mu_{\ln \xi(\tilde{X})}\right)
$$

where $\xi(\tilde{X})$ is the respective soil property assigned to the different zones $(\tilde{X})$ in the problem domain, $\varepsilon_{\ln \xi(\tilde{X})}$ is an independent standard normally distributed random field with zero mean and unit variance, $\mu_{\ln \xi(\tilde{X})}$ is the mean of the logarithm of $\xi$ and $L$ is a lower-triangular matrix which is obtained from the covariance matrix using Cholesky decomposition technique. For an isotropic soil stratum, the covariance matrix is defined as a general exponential Markovian function as follows:

$$
A(d)=\sigma_{\ln \xi}^{2} \exp \left(-\frac{2|d|}{\theta}\right)
$$

where "d" is the center to center distance of two zones and $\sigma_{\ln \xi}^{2}$ is the variance of the logarithm of $\xi . \mu_{\ln \xi(\tilde{X})}$ (in Eq. 8) and $\sigma_{\ln \xi}^{2}$ (in Eq. 9) are obtained adopting log-normal distribution transformations as follow:

$$
\begin{aligned}
& \mu_{\ln \xi}=\ln \left(\mu_{\xi}\right)-\frac{1}{2} \sigma_{\ln \xi}^{2} \\
& \sigma_{\ln \xi}^{2}=\ln \left(1+\sigma_{\xi}^{2} / \mu_{\xi}^{2}\right)=\ln \left(1+C O V_{\xi}^{2}\right)
\end{aligned}
$$

Using the Cholesky decomposition technique, the covariance matrix A, defined by Eq. 9, can be split into two matrices as follows:

$$
A=L L^{T}
$$

where $\mathrm{L}^{\mathrm{T}}$ is the transpose matrix of T. Matrix L is then substituted in Eq. 8 to thoroughly characterize the random soil property $\xi$.

\section{Reconstitution of heterogeneous samples}

As mentioned earlier, in the second part of this study, nine soil clusters were mixed to form stochastic realizations with the moisture contents corresponding to their respective liquid limits. This amount of moisture content is sufficient to achieve a level of workability suitable to make the strings of slurry in the heterogeneous sample preparation process. The moisture content of the mixtures could not be enhanced further due to the high compressibility of the slurry, which in turn would not leave enough solid material during consolidation and upon shearing. Adopting different soil clusters along with the random field theory concepts discussed above, several heterogeneous soil physical models were constructed in this study. Figure 11 shows the different stages of heterogeneous physical model construction and its subsequent consolidation.

In total, six heterogeneous models with different stochastic parameters were constructed and tested in this study. Each heterogeneous soil model consists of one hundred elements of the random field placed one by one in layers starting from the bottom of the box. The basic properties of the six physical models along with their stochastic 

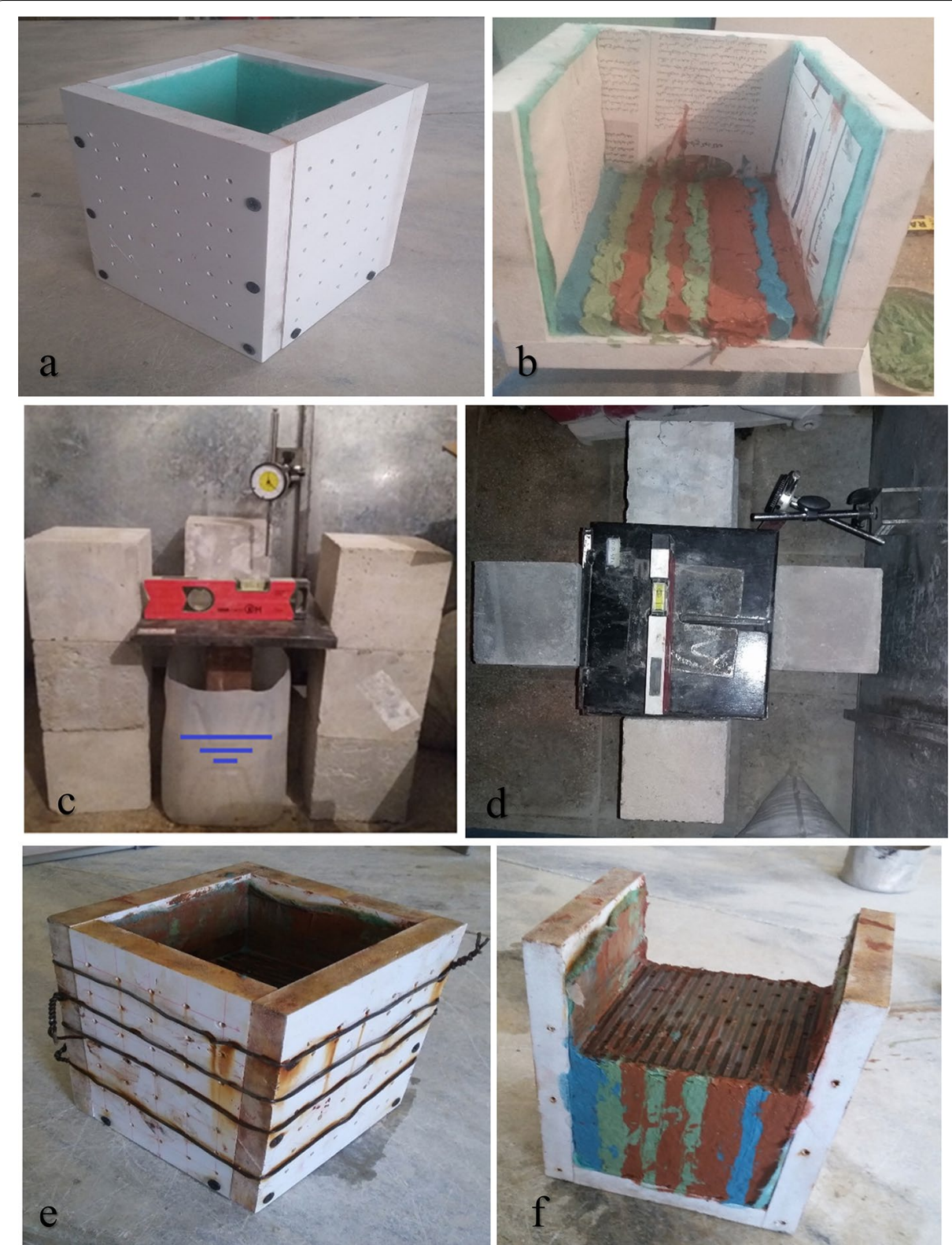

Fig. 11 Heterogeneous soil physical model construction and subsequent consolidation

parameters, including the coefficient of variation $(\mathrm{COV})$ and the scale of fluctuation $\left(\theta_{\mathrm{v}}\right)$, are summarized in Table 4 . The heterogeneous physical model was constructed by using the syringe for placing the strings of slurries in the prefabricated perforated wooden box, which was $150 \mathrm{~mm}$ deep, $100 \mathrm{~mm}$ wide and $100 \mathrm{~mm}$ long (Fig. 11). For drainage purposes, several holes have been embedded on the side walls and bottom plates of the box which were covered with some filter sheets to prevent the holes from getting clogged. In order to restrict the model laterally, the box was tied up with a metal wire.

Once the heterogeneous sample was constructed, it was subjected to consolidation. All the samples were consolidated in three different overburden pressures, including 
Table 4 Specifications of heterogeneous soil physical models

\begin{tabular}{lllllll}
\hline Sample no. & COV & $\boldsymbol{\theta}_{\mathbf{v}}(\mathbf{c m})$ & Density $\left(\mathbf{g} / \mathbf{c m}^{\mathbf{3}}\right)$ & $\begin{array}{l}\text { Initial void } \\
\text { ratio, } \boldsymbol{e}_{\boldsymbol{o}}\end{array}$ & $\begin{array}{l}\text { Compression } \\
\text { index, } \boldsymbol{C}_{\boldsymbol{c}}\end{array}$ & $\boldsymbol{c}_{\boldsymbol{v}}\left(\mathbf{m}^{\mathbf{2}} \mathbf{/ s}\right)^{\mathbf{a}}$ \\
\hline $\mathrm{HS}-1$ & 0.25 & 2 & 1.55 & 4.51 & 1.77 & $1.30885 \times 10^{-9}$ \\
$\mathrm{HS}-2$ & 0.5 & 2 & 1.59 & 3.59 & 1.61 & $6.07592 \times 10^{-9}$ \\
$\mathrm{HS}-3$ & 0.75 & 2 & 1.58 & 3.36 & 1.68 & $1.72791 \times 10^{-8}$ \\
$\mathrm{HS}-4$ & 0.25 & 20 & 1.51 & 4.44 & 1.69 & $1.22319 \times 10^{-9}$ \\
$\mathrm{HS}-5$ & 0.5 & 20 & 1.51 & 3.69 & 1.56 & $1.31596 \times 10^{-9}$ \\
$\mathrm{HS}-6$ & 0.75 & 20 & 1.54 & 3.36 & 1.41 & $1.50195 \times 10^{-9}$ \\
\hline
\end{tabular}

a Coefficient of consolidation at pressure $100 \mathrm{kPa}$
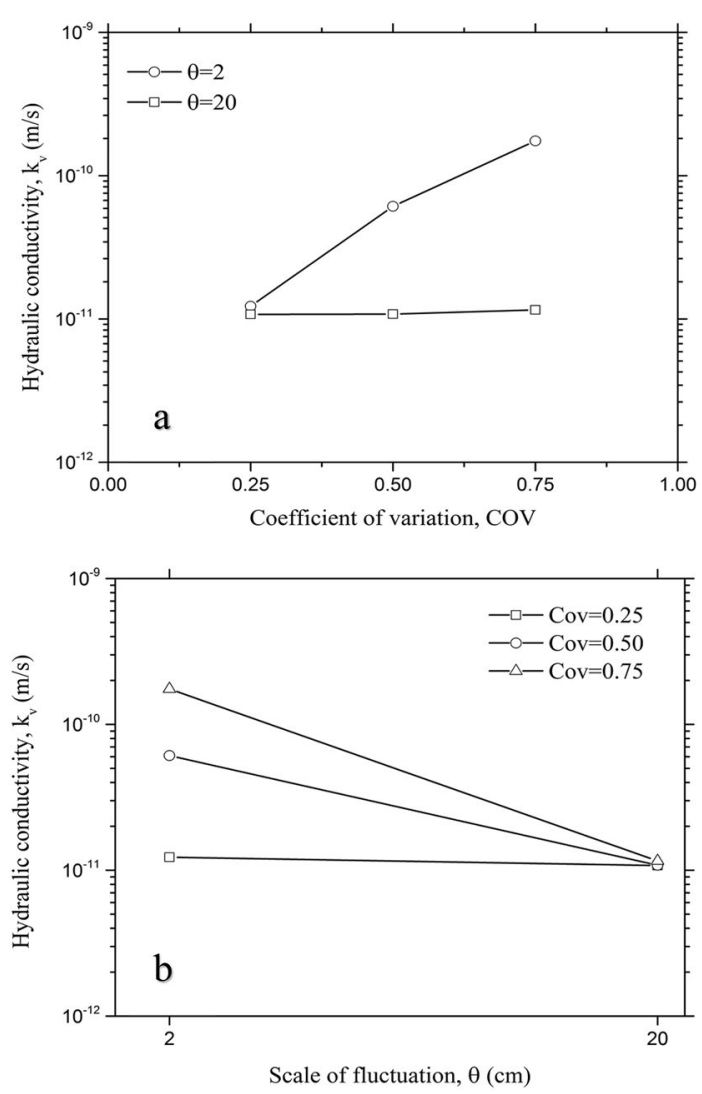

Fig. 12 Variation of the coefficient of permeability with: a coefficient of variation; and $\mathbf{b}$ scale of fluctuation for heterogeneous soils

$25 \mathrm{kPa}, 50 \mathrm{kPa}$ and $100 \mathrm{kPa}$. Each pressure step was maintained for $48 \mathrm{~h}$ to allow for the dissipation of the excess pore water pressure.

\section{Hydraulic conductivity of the heterogeneous models}

During the process of consolidation for the prepared heterogeneous physical models, their settlements were recorded at the end of each equilibrium stage. Table 4 shows the measured compressibility parameters of the heterogeneous tested samples. Based on the values presented in this table, the coefficient of consolidation increases, while the compression index decreases with the increase of the coefficient of variation (COV). 


\begin{tabular}{|c|c|c|c|c|c|}
\hline \multirow[t]{2}{*}{ Designation } & \multicolumn{2}{|c|}{ Homogeneous clusters } & \multirow[t]{2}{*}{ Designation } & \multicolumn{2}{|c|}{ Heterogeneous samples } \\
\hline & $k_{v}(m / s)$ & $m_{v}\left(m^{2} / k N\right)$ & & $k_{v}(m / s)$ & $m_{v}\left(m^{2} / k N\right)$ \\
\hline S-1 & $2.09076 \times 10^{-9}$ & $3.44 \times 10^{-4}$ & HS-1 & $1.22833 \times 10^{-11}$ & 0.00095666 \\
\hline S-2 & $9.98101 \times 10^{-11}$ & $4.68 \times 10^{-4}$ & HS-2 & $6.11587 \times 10^{-11}$ & 0.00102607 \\
\hline$S-3$ & $1.78764 \times 10^{-11}$ & $7.26 \times 10^{-4}$ & HS-3 & $1.74753 \times 10^{-10}$ & 0.00103094 \\
\hline S-4 & $1.05773 \times 10^{-11}$ & $7.54 \times 10^{-4}$ & HS-4 & $1.07687 \times 10^{-11}$ & 0.00089743 \\
\hline S-5 & $1.04106 \times 10^{-11}$ & $7.69 \times 10^{-4}$ & HS-5 & $1.08087 \times 10^{-11}$ & 0.00083726 \\
\hline S-6 & $1.081 \times 10^{-11}$ & $9.26 \times 10^{-4}$ & HS-6 & $1.15716 \times 10^{-11}$ & 0.00078536 \\
\hline S-7 & $1.23047 \times 10^{-11}$ & $1.13 \times 10^{-3}$ & & & \\
\hline S-8 & $1.12702 \times 10^{-11}$ & $1.85 \times 10^{-3}$ & & & \\
\hline S-9 & $1.01661 \times 10^{-11}$ & $2.41 \times 10^{-3}$ & & & \\
\hline
\end{tabular}

Figure 12 shows the variation of the coefficient of permeability with stochastic parameters for heterogeneous soil samples. The higher coefficient of variation for heterogeneous soils is an indication of more variability of the mixtures. Based on the experimental results depicted in Fig. 12a, the coefficient of permeability increases with the increase of the coefficient of variation. Moreover, increasing the scale of fluctuation from 2 to $20 \mathrm{~cm}$ leads to the lower obtained values for the permeability coefficient and coefficient of consolidation, as illustrated in Fig. 12b.

Table 5 presents the comparison between the compressibility parameters for homogeneous soil mixtures and heterogeneous soil physical models. The permeability coefficients for the heterogeneous soils are close to the pure bentonite due to the threshold bentonite content discussed earlier. Also sample HS-3 has the highest permeability coefficient values due to the high percentage of kaolin present in these soil models.

\section{Concluding remarks}

In this study, the physical modelling of heterogeneity was substantiated by combining nine homogeneous soil clusters of kaolin-bentonite mixtures using the random field theory concepts. Considering the preliminary properties of the kaolin-bentonite mixtures and adopting variable specific realizations of their liquid limits, different discrete cells in the heterogeneous physical model were associated with the particular random soil properties. The constructed physical models were then subjected to successive consolidation stages in a standard oedometer apparatus and the respective consolidation parameters were quantified. Based on the experimental results, soil heterogeneity and inherent variability were observed to have a considerable influence on the consolidation parameters of the soils. Furthermore, the results showed that the greater heterogeneity in the soils, expressed through higher coefficient of variation (COV), leads to the increase in the hydraulic conductivity, while the larger correlation distance inside the heterogeneous physical model results in the increase in the time required for the excess pore water pressure to dissipate, which in turn leads to the reduction of the hydraulic conductivity and coefficient of consolidation. The experimental modelling of soil spatial variability, developed in the past studies and employed here, could be utilized as a verification tool for the numerical simulation of random field theory. However, more 
justifications are required on the minimum number of physical realizations to capture a vivid picture of the stochastic variation of mechanical and hydraulic parameters of heterogeneous samples in geotechnical engineering applications.

\section{Authors' contributions}

RJC supervised this study in forms of two MSc theses. He also served as the main contributor of this paper by substantially revising the manuscript both technically and editorially. AT carried out the heterogeneous consolidation experiments and analyzed the experimental data accordingly. MG contributed to the manuscript preparation by delivering the first draft. MP was an advisor of these research tasks and he provided an excellent revision to this manuscript. All authors read and approved the final manuscript.

\section{Author details}

${ }^{1}$ Department of Civil Engineering, Faculty of Engineering, University of Guilan, P.O. 3756, Rasht, Guilan, Iran. ${ }^{2}$ Department of Civil Engineering, Shahid Rajaee Teacher Training University, Lavizan, Tehran, Iran.

\section{Competing interests}

The authors declare that they have no competing interests.

\section{Publisher's Note}

Springer Nature remains neutral with regard to jurisdictional claims in published maps and institutional affiliations.

Received: 7 June 2018 Accepted: 2 December 2018

Published online: 10 December 2018

\section{References}

1. ASTM, D2435-03 (2003) Standard test method for one-dimensional consolidation properties of soils. Annual Book of ASTM standards

2. Baecher GB, Ingra TS (1981) Stochastic FEM in settlement predictions. J Geotech Eng 107(4):449-463

3. Burland JB (1990) On the compressibility and shear strength of natural clays. Géotechnique 40(3):329-378

4. Cho SE, Park HC (2009) Effect of spatial variability of cross-correlated soil properties on bearing capacity of strip footing. Int J Num Anal Methods Geomech 34(1):1-26

5. Dakshanamurthy $V$, Raman V (1973) A simple method of identifying an expansive soil. Soils Found 13(1):97-104

6. Fenton GA, Griffiths DV (2002) Probabilistic foundation settlement on spatially random soil. J Geotech Geoenviron Eng 128(5):381-390

7. Fenton GA, Griffiths DV (2003) Bearing capacity prediction of spatially random c-u soils. Can Geotech J 40(1):54-65

8. Fenton GA, Griffiths DV (2008) Risk assessment in geotechnical engineering. Wiley, New York

9. Garzón LX, Caicedo B, Sánchez-Silva M, Phoon KK (2015) Physical modelling of soil uncertainty. Int J Phys Model Geotech 15(1):19-34

10. Ghoreishi M (2017) Numerical modelling of uncertainty in shear load-displacement characteristics of cohesive soils using FLAC 3D. In: Partial fulfillment of the requirements the degree of Master of Science. Faculty of Engineering, University of Guilan, Guilan

11. Griffiths DV, Fenton GA (2001) Bearing capacity of spatially random soil: the undrained clay Prandtl problem revisited. Geotechnique 51(4):351-359

12. Haldar S, Babu Sivakumar GL (2007) Effect of soil spatial variability on the response of laterally loaded pile in undrained clay. Comput Geotech 35:537-547

13. Hicks MA, Samy K (2002) Influence of heterogeneity on undrained clay SLOPE stability. Q J Eng Geol Hydrogeol 35(1):41-49

14. Jamshidi Chenari R, Alaie R (2015) Effects of anisotropy in correlation structure on the stability of an undrained clay slope. Georisk 9(2):109-123

15. Jamshidi Chenari R, Zamanzadeh M (2016) Uncertainty assessment of critical excavation depth of vertical unsupported cuts in undrained clay using random field theorem. Int J Sci Technol (Scientia Iranica) 23(3):864-875

16. Jamshidi Chenari R, Ghorbani A, Eslami A, Mirabbasi F (2018) Behavior of piled raft foundation on heterogeneous clay deposits using random field theory. Civil Eng Infrastruct J 51(1):35-54. https://doi.org/10.7508/ceij.2018.01.003

17. Papoulis D, Tsolis-Katagas P, Katagas C (2004) Progressive stages in the formation of Kaolin minerals of different morphologies in the weathering of Plagioclase. Clays Clays Min 52(3):275-286

18. Ranjbar Pouya K, Zhalehjoo N, Jamshidi Chenari R (2014) Influence of random heterogeneity of cross-correlated strength parameters on bearing capacity of shallow foundations. Indian Geotech J 54(4):427-435

19. Sivakumar Babu GL, Mukesh MD (2004) Effect of soil variability on reliability of soil slopes. Geotechnique 54:335-337

20. Srivastava A, Sivakumar Babu GL (2009) Effects of soil variability on the bearing capacity of clay and in slope stability problems. Eng Geol 108:142-152

21. Taleb SA (2017) Physical modelling of uncertainty in deformation characteristics of soils. In: partial fulfillment of the requirements the degree of Master of Science. Faculty of Engineering, University of Guilan, Guilan

22. Zhalehjoo N, Jamshidi Chenari R, Ranjbar Pouya K (2012) Evaluation of bearing capacity of shallow foundations using random field theory in comparison to classic methods. In: Proceedings of GeoCongress 2012: state of the art and practice in geotechnical engineering. Oakland, California, pp 2971-2980 